www.pasosonline.org

\title{
Los Organismos de Gestión de Destino en Enoturismo: Casos Internacionales de Éxito ${ }^{[a]}$
}

\author{
Alba Fernández Alonso* \\ Tourism Innovation Lab \\ Lola Herrero Amo** \\ ESCP Europe (Madrid Campus) \\ José Antonio Vidal*** \\ Asociación Española de Enoturismo (España)
}

\begin{abstract}
Resumen: La actividad enoturística ha evolucionado en España de forma más lenta que en otras zonas del mundo, entre otras causas por una gestión de destinos de menor éxito en su implementación. Por ello resulta esencial identificar y analizar casos de éxito enoturístico en organismos de gestión de destinos (OGDs) a nivel internacional, permitiendo formular un conjunto de recomendaciones potencialmente extrapolables a destinos enoturísticos españoles. En un ejercicio de benchmarking se mapea una tríada de variables relevantes. En primer lugar, las variables culturales ("Viejo Mundo" y "Nuevo Mundo"); luego, los distintos niveles administrativos de gestión (nacional-regional-local); y en tercer lugar, el aspecto geográfico, con análisis de casos en tres continentes: Francia, Condado de Sonoma (California, EEUU), y Cabo Wineland (Sudáfrica). Con este acopio de datos y un análisis de la situación del enoturismo en España se formulan algunas propuestas de mejora potencialmente aplicables a la gobernanza y gestión en destinos enoturísticos españoles.
\end{abstract}

Palabras Clave: Enoturismo. Organismos de Gestión de Destino (OGDs). Casos de éxito en enoturismo. Recomendaciones de política turística y gobernanza.

\section{Wine tourism and destination managementorganizations: international case studies $^{[a]}$}

Abstract: Wine tourism has developed in Spain at a slower rate than elsewhere, because of a less successful destination management approach among other reasons. In this framework, it is relevant to identify and analyse successful wine tourism destinations worldwide, as their policy and governance practice may be inspiring even for destination management organizations and entrepreneurs in Spain. A benchmarking exercise is thus carried out - taking into consideration cultural and institutional variables as well as geolocation characteristics - for case studies in France, Sonoma County (California, U.S.A.) and Cape Wineland (South Africa). A comparison of best practices there and for wine tourism (enotourism) in Spain permits to enunciate some conclusions and recommendations.

Keywords: Wine tourism. Destination Management Organizations. Successful cases in wine tourism. Tourism policy and governance recommendations.

* Tourism Innovation Lab (t-Lab) albafalonso@tourisminnovationlab.org

** École Supérieure de Commerce de Paris (ESCP Europe) lherrero@escpeurope.eu

*** Asociación Española de Enoturismo (AEE) j.vidal@aee-gob.org

[a] Los autores desean expresar su reconocimiento al Prof. Dr. Eduardo Fayos-Solà, George Washington University, (edsola@ gwu.edu), por sus sugerencias metodológicas, indicaciones y supervisión.

[a] The Authors wish to acknowledge with thanks the support of Prof.Eduardo Fayos-Solà, George Washington University, (edsola@gwu.edu), in providing with methodological guidance as well as suggestions and final supervision. 


\section{Introducción}

En el contexto de las tendencias que se vienen imponiendo en el sector turístico ligadas a la fragmentación de los periodos vacacionales (Fayos-Solà et al, 2012a), la híper-segmentación del producto turístico o el auge del turismo experiencial (López-Guzmán, Rodríguez y Vieira, 2013), el enoturismo se posiciona como un componente fundamental del turismo gastronómico (OMT, 2016a), que por su conceptualización, basada en los elementos tangibles e intangibles desarrollados en torno al vino en sí mismo, supone un exponente del turismo sensorial (Getz, 2000) y vivencial y un factor de desarrollo de áreas eminentemente rurales (OMT, 2016a; López-Guzmán et al, 2013; Vázquez de la Torre y Melián, 2008; Carlsen y Charters, 2006; Williams, Graham y Mathias, 2006).

La actividad enoturística empezó lentamente su expansión en España en la década de 1990 y el análisis de su desarrollo refleja que su crecimiento se ha visto ralentizado frente a otras regiones o destinos internacionales, con tendencia a estancarse y acomodarse (Cabello y Pascual, 2015), fenómeno paralelo con los problemas de transición del mercado vitivinícola (Gatti, Giraud-Héraud y Mili, 2003). Ello se ha debido a diferentes factores, entre los que cabría destacar: (i) la falta de comunicación entre los diferentes "actores" públicos y privados que participan en esta actividad; (ii) los diferentes niveles de conocimiento, identidad o diferenciación de los destinos enoturísticos, más enfocados a "la restructuración de las economías rurales" (Ramis, 2010), sesgo endémico del Viejo Mundo; y (iii) la necesidad de "profesionalización" de los diferentes actores que participan en el desarrollo del enoturismo, mencionada siempre como desiderátum futuro por diversos autores (Elías, 2006).A pesar de ello se observa un potencial de crecimiento del enoturismo en destinos turísticos españoles con cifras que alcanzan los más de 2,5 millones de enoturistas, considerando únicamente las Rutas del Vino en 2016, suponiendo un aumento en términos absolutos del 21\% respecto al año anterior (Acevin, 2017), y con la aparición de nuevos actores estratégicos en el panorama nacional, como la Asociación Española de Enoturismo.

Según datos de la Federación Española del Vino (FEV, 2017) y del Observatorio Español del Mercado del Vino (OEMV, 2017a), España es uno de los líderes mundiales en exportación de vino, con un importante crecimiento fuera de la Unión Europea, en especial hacia Estados Unidos y China. Es, con casi 4.000 millones de hectolitros, el tercer productor mundial de vino tras Italia y Francia, y por delante de países como Estados Unidos, Australia, China o Sudáfrica (OIV, 2017). Además posee la superficie de viñedo más importante del mundo (975.000 ha. según datos de la Organización Internacional de la Viña y el Vino, lo que supone un 14\% de la superficie mundial (OEMV, 2017b), y cuenta con más de 4.000 bodegas que se ubican en 130 regiones vinícolas oficiales (OEMV, 2017c).

Para alcanzar el desarrollo enoturístico máximo en España conviene examinar modelos creados por Organismos de Gestión de Destinos (OGDs o DMOs - Destination Management Organizations - por sus siglas en inglés) que hayan tenido éxito en el ámbito internacional, lo que permitirá formular un conjunto de recomendaciones potencialmente extrapolables al modelo español (Fayos-Solà, García y Moreda, 2002). Como se indicaba en el Congreso "Enoturismo, una estrategia para todos" (2015) se trataría de ver "qué es lo mejor de cada zona y ofrecer alternativas al enoturista" (Morgenthal, 2015).

La experiencia demuestra que se puede compartir conocimientos y buenas prácticas (Fayos-Solà, Alvarez y Cooper, 2014; Sigala, Christou y Gretzel, 2012; Christou y Nella, 2012; Alebaki y Iakovidou, 2011), trabajando en soluciones sobre problemas similares alrededor del mundo (benchmarking). De esta forma, el propósito de este trabajo es identificar las mejores prácticas de la función de OGDs en enoturismo internacional, con el objetivo de identificar un abanico de propuestas que contribuyan a la mejora de la gestión de los destinos enoturísticos en España:

- ¿Cuáles son los casos de éxito a nivel internacional en la gestión enoturística?

- ¿Qué modelos de Organismos de Gestión de Destino (OGDs/DMOs) se están imponiendo y qué políticas enoturísticas se implementan?

- ¿Cuál es el estado de la cuestión del enoturismo en España?

No obstante, antes de proceder a la revisión de modelos de éxito internacionales, y al estado de la cuestión de este fenómeno en España, y con el fin de presentar adecuadamente los resultados obtenidos y las propuestas derivadas de este análisis, parece necesario un breve acercamiento al marco referencial-conceptual, sobre los conceptos claves definitorios del enoturismo 


\section{Marco referencial: enoturismo y OGDS}

\section{1 ¿Qué información se maneja sobre la función de los OGDs en destinos enoturísticos?}

La gestión del destino turístico, desde la conceptualización de la experiencia turística entendida como satisfacción de la expectativa de una manera global, tiene en cuenta la alineación de diferentes elementos de la oferta, integrando bienes y servicios, recursos, infraestructuras y equipamientos, planificación y gestión, imagen de marca y precio, y que han de sumarse al componente motivacional que todo destino ofrece (Herrero, 2014).

Tal cúmulo de componentes, su complejidad, y las posibilidades de resolución de los conflictos derivados de los múltiples y diferentes intereses de los actores que intervienen en el sistema turístico, requieren de una metodología orientada al diseño de estrategias adaptadas a las realidades locales de esos actores. Realidades que han de propiciar la formación de redes de coordinación y colaboración entre los diferentes actores públicos y privados (Muñoz y Velasco, 2015). Es en esa colaboración donde se pone de manifiesto el fortalecimiento de los servicios a través de la constitución de entidades de carácter superior (OGDs) que incrementan la ventaja competitiva que los actores (empresas fundamentalmente) no pueden adquirir de manera individual (Cosa, 2010).

En el caso específico del enoturismo en España - que se ha intentado desarrollar a partir de rutas turísticas que ofrecen diversos recursos y atractivos (naturales, físicos, culturales, gastronómicos...) esa alianza público-privada tiene el triple objetivo de impulsar, dirigir y dinamizar el producto/destino turístico (López-Guzmán y Sánchez, 2008). Un triple objetivo que puede ser concretado, siguiendo a Díaz (2008), como una estrategia donde desarrollar un modelo enoturístico orientado a la comercialización del destino ante los mercados, al mismo tiempo que se posibilita la coordinación interna (local) de los recursos de las empresas involucradas.

En la práctica, se traduce en la ejecución de otras actividades tales como la gestión de flujos; análisis de la demanda; organización de la oferta turística; desarrollo de nuevos productos; adopción de adecuadas políticas de precio y distribución; y la selección de segmentos de mercado a los que dirigirse y que podrían estar interesados en el destino (Cosa, 2010:15)

El concepto de OGD ha evolucionado con el tiempo desde una perspectiva de marketing (Destination Marketing Organization) a una perspectiva de gestión (Destination Management Organization), como señalan Sheehan et al. (2016). Según la OMT (2007:14), las OGDs/DMOs suponen la estructura de base para la gestión de destinos turísticos:

La gestión de destinos exige una coalición de muchas organizaciones e intereses que trabajan hacia un objetivo común. El papel de la Organización de Gestión de Destino (DMO) debe ser dirigir y coordinar actividades bajo una estrategia coherente. No controlan la actividad de sus socios, sino que reúnen los recursos y la experiencia y el grado de independencia y objetividad para guiar el camino. Se deduce que las DMOs deben desarrollar un alto nivel de habilidades en el desarrollo y gestión de asociaciones. Aunque las organizaciones de gestión de destinos han llevado a cabo típicamente actividades de mercadotecnia, su mandato se está volviendo mucho más amplio, para convertirse en un liderazgo estratégico en el desarrollo del destino.

Sin embargo, y pese a la importancia teórica de la planificación y gestión, ya mencionada por diversos autores como aspecto clave en el estado de la cuestión enoturística (Charters y Carlsen, 2006; López-Guzmán, Rodríguez, y Vieira, 2013), y pese a la aproximación a los OGDs como estructura básica para el desarrollo de los destinos - tanto en las regiones enoturísticas del Viejo como en las del Nuevo Mundo - parece que el enoturismo no es una prioridad. Como señala Loubser (2004):

- A nivel regional, los gobiernos no consideran el turismo enológico como una prioridad real y se centran en su propia estrategia de crecimiento a través de otras industrias.

- Se observa una descoordinación entre los distintos actores de la actividad enoturística.

- Asimismo, las inversiones en enoturismo rara vez resultan de estrategias conscientemente formuladas y basadas en información fiable y actualizada. Los datos a menudo no se registran, o cuando son registrados, no se analizan para la toma de decisiones. De esta manera, los esfuerzos de investigación e inteligencia de mercado - si los hay- tienden a dispersarse. 
Sin embargo, aunque no de manera "articulada" a través de una estructura de OGDs, sí aparecen algunos ejemplos de grandes inversiones por parte de fincas y bodegas para atraer clientes a sus propuestas de enoturismo.

\section{2 ¿Cómo se conceptualiza el enoturismo y la demanda enoturística?}

En la conceptualización del enoturismo aparece sistemáticamente un enfoque holístico y multisectorial de los OGDs/DMOs. Maduro, Guerreiro y Oliveira (2015: 5) lo definen como "un conjunto de servicios turísticos, actividades de ocio y tiempo libre dedicado al descubrimiento y placer cultural y enológico de la vid y el vino", mientras que la Asociación Española de Enoturismo (AEE) ha propuesto a la Real Academia Española de la Lengua (RAE) la definición de enoturismo como "Modalidad de turismo basada en desplazamientos a entornos vinícolas, con el propósito de conocer, disfrutar y compartir experiencias en torno a la Cultura del Vino" (AEE, 2015).

La Red Europea de Ciudades del Vino (Recevin) realizó, dentro del proyecto europeo Interreg IIIc (2004-2006), el proyecto "Vintur: el espacio europeo del enoturismo" que supuso un ejemplo de este enfoque holístico. En su documento "Vademecum del Enoturismo Europeo" (Recevin, 2006) incorpora diferentes ideas, entre las que destaca el valor enológico del producto (experiencia) enoturístico, es decir, el enoturismo no existe sin la cultura del vino. En este sentido, la cultura del vino es el eje temático de este producto/experiencia y el turista debe ser capaz de percibirlo durante todas las etapas de su viaje, independientemente del lugar que el enoturismo ocupe en la cadena de valor del destino turístico.

Las evidencias encontradas en el estudio de la estructura y el comportamiento de la demanda enoturística (vid. infra, sección 4) y el enfoque de López-Guzmán et al (2013: 172) sobre el "interés creciente por todo lo relacionado con el vino [...] la gastronomía, el turismo, la cultura y factores adyacentes..." - nos indican que el enoturista busca una experiencia cultural total. Ello está en consonancia con la definición de enoturismo como aquella modalidad de turismo que gira en torno a la bodega como elemento dinamizador. En el otro lado están los viticultores, más interesados en las ventas de vino, tanto en sus bodegas como fuera de ellas.

Todo ello indica claramente una oportunidad de mejora en la sensibilización de los productores de vino, ya señalada por el anteriormente citado Loubser (2004). Esta sensibilización y capacitación de los productores debería estar basada en tres ejes: (i) la comprensión del enoturismo como una experiencia/ cadena de valor; (ii) el diseño de productos/experiencias enoturísticas basadas en la co-creación (enfatizando los "requisitos de servicio"); y (iii) la sostenibilidad y mejora de la comercialización del enoturismo.

En esta línea se mueve también López-Sánchez (2010: 39) quien, analizando el sistema turístico de las principales "Rutas del Vino" en España, apunta que este sistema ha de basarse en cinco elementos clave: (i) la planificación y la gestión del producto; (ii) la profesionalización de la gestión basada en la innovación; (iii) el desarrollo de campañas de marketing basadas en la especificidad de los destinos mediante la puesta en valor de la identidad cultural vitivinícola de las regiones; (iv) el diseño de sistemas de mejora continua que garanticen la calidad del producto; y por último (v) la formación continua de los profesionales encargados del desarrollo turístico.

\section{Analisis de la función de los OGDS en Enoturismo: una aproximación a casos internacio- nales de éxito}

A partir de un mapeo/benchmarking internacional de las funciones de los OGD/DMOs en enoturismo, se presentan a continuación ejemplos de buenas prácticas identificadas en tres destinos enoturísticos de referencia: Francia, el Condado de Sonoma (California, EEUU) y Cabo Wineland (Sudáfrica).

\subsection{El caso de Francia: Visit French Wine}

Tres millones de personas visitaron Francia en 2010 con el enoturismo como motivo principal de su viaje (Domenach, 2016). La tendencia creció rápidamente y en 2016 fueron diez millones de clientes los que visitaron instalaciones enoturísticas en Francia (con un 58\% de visitantes domésticos y un $42 \%$ de turistas internacionales), realizando un gasto de más de 5,2 mil millones de euros (VFW, 2017). La mayor parte de los turistas internacionales procedían de Bélgica, Reino Unido, Países Bajos y Alemania.

Debido al crecimiento en el número de visitantes, varias organizaciones del sector público y privado trabajan en el desarrollo, promoción y gestión del enoturismo en Francia. Destacan Atout France y FranceAgriMer, organizaciones del sector público que trabajan con el objetivo de crear un fuerte desar- 
rollo económico de la industria vitivinícola a través de diferentes acciones (marketing, colaboraciones, promoción, etc.).

Atout France está al frente de la entidad especializada en enoturismo Visit French Wine. Este organismo de promoción (en forma de OGD) fue constituido con el apoyo del Ministerio de Asuntos Exteriores de Francia en febrero de 2016 y tiene como objetivo principal atraer enoturistas potenciales a través de información útil que destaca diferentes "recursos", en torno a la cultura del vino. Diseña acciones de comunicación y promoción basadas en personas clave (líderes de opinión) en enoturismo, productos y productores (bodegas), ofertas, negocios y eventos relacionados con el vino. Es por tanto una entidad especializada en el desarrollo específico del enoturismo, pero con una vinculación estratégica esencial con Atout France (cuyo principal objetivo es contribuir al desarrollo de la actividad turística en Francia). Ambas organizaciones actúan en el contexto de la misma misión, siendo el desarrollo del enoturismo el único objetivo de Visit French Wine, entre cuyas tareas se incluyen:

- Observación y análisis de los mercados enoturísticos, para definir una estrategia que contribuya a optimizar las opciones de inversión en materia de promoción, comunicación y estructura de asociaciones con los canales de distribución.

- Diseño y comercialización de las ofertas turísticas francesas en enoturismo (producto). Esta tarea tiene como objetivo aumentar la competitividad de los productos franceses y ayudar a sus productores en la promoción a escala mundial.

- Optimización de la calidad de los productos y servicios relacionados con el enoturismo, gracias a la gestión de una clasificación de alojamientos franceses, registro de operadores y promoción de etiquetas como "Bodegas y Descubrimientos" para el enoturismo.

- Intervención local, nacional e internacional, gracias a las 32 oficinas en 27 países de Atout France.

Para cumplir con sus objetivos principales, Visit French Wine implementa diferentes líneas de trabajo. Por ejemplo, ha creado una entidad asociativa específica, con la participación de otras instituciones especializados y vinculadas a la industria del vino como empresas locales de distintos sectores, bodegas y OGDs locales especializados en la industria del vino. Estas colaboraciones público-privadas incluyen la asociación con stakeholders (guías, chefs, museos, restaurantes, bodegas, viñedos y asociaciones) permitiendo la integración y coordinación de todos los participantes de la industria enoturística.

Respecto a su línea de acción con la demanda, a través de la plataforma de Visit French Wine los enoturistas tienen diferentes opciones para acceder a la información que necesitan. Mediante una web acceden a un mapa con los diferentes destinos turísticos y una vez seleccionado alguno de ellos aparece información específica en "hojas de identidad" para cada región vitivinícola de Francia. Luego, cuando los clientes potenciales acceden a estas "hojas de identidad", obtienen información sobre la región y su producción de vino, las razones para ir a ese destino, enlaces a otros sitios web y videos, ideas de actividades, consejos de expertos, noticias y eventos.

Otra opción, más personalizada, pasa por cumplimentar un breve cuestionario de tres sencillas preguntas, lo que les permite obtener una propuesta enoturística personalizada y adaptada a sus necesidades.

De cualquier forma y en ambas opciones de búsqueda en la plataforma Visit French Wine, se promueven experiencias - por ejemplo a través de la descripción de catas de vino / tours, propuestas de restauración, eventos, celebración y descubrimiento del vino de una región, ferias y testimonios - que ayudan a los clientes a encontrar una "experiencia enoturística" adaptada a sus necesidades.

Cuando un cliente potencial encuentra la información que necesita es redirigido al sitio web oficial de los proveedores para ponerse en contacto directo con ellos y reservar directamente. Este enfoque facilita sin duda el hecho de que el $93 \%$ de la reserva registrada de experiencias y productos enoturísticos se realizó en canales directos en 2016.

No hay ventas directas en VisitFrenchWine.fr, ya que su único objetivo es promover Francia como un destino enoturístico, de manera que los diferentes actores y prestatarios de servicio comparten información con la plataforma y ésta se ocupa de la promoción, ejerciendo por tanto funciones propias de una entidad de marketing de destino.

Los diferentes actores de cada región trabajan juntos para promover sus recursos y productos, y transmitir autenticidad con el fin de atraer a nuevos clientes. Para tener un impacto más amplio y una mejor visibilidad, Visit French Wine está presente en todos los medios sociales como Twitter, Facebook, Instagram, Pinterest y Google Plus. Por otra parte, muchos artículos hablan de la plataforma y sus utilidades aumentando su visibilidad. 


\subsection{El caso de Sudáfrica: WOSA (Wines of South Africa) y Ruta del Vino de Stellenbosch}

El desarrollo de productos enoturísticos supone uno de los recursos turísticos más importantes en Sudáfrica, por lo que el enoturismo se ha convertido en uno de los pilares más importante en las estrategias globales de destino de la última década. Este país se situaba ya en 2013 entre los 10 principales destinos de turismo del vino en todo el mundo según blogs especializados como Viator (2013). Sudáfrica se sitúa entre los primeros ocho productores mundiales de vino (WOSA, 2016), si bien por detrás de España. El éxito de Sudáfrica se ha producido gracias a una apuesta estratégica conjunta, tanto a nivel nacional como internacional. La directora ejecutiva de WOSA, Sue Birch (2007), explicó en Vinexpo Bordeaux 2007 las razones de dicho éxito, con una estrategia basada en el denominado "enfoque colectivo del enoturismo". Dicho enfoque ha permitido la creación de 18 clusters de vino diferentes basados en características únicas y específicas, en la región de Cabo Occidental. Esta experiencia de desarrollo colectivo muestra que las bodegas no podrían, de forma aislada e independiente, comercializar una región enoturística: es necesaria una formulación de objetivos comunes y recursos operacionales como base del éxito de la estrategia de desarrollo enoturístico en Sudáfrica.

WOSA, establecida en 1999, ha sido la impulsora de esta estrategia. Se trata de una entidad independiente de cualquier organismo gubernamental (si bien reconocida oficialmente), empresa mayorista o productor (bodega). WOSA es por tanto una estructura inspirada en las OGDs/DMOs, si bien más cercana al concepto de organismo de marketing de destino (destination marketing organization), muy especializada y encargada de promover únicamente las regiones vitivinícolas del país, contribuyendo a la exportación de los vinos sudafricanos en los principales mercados internacionales.

WOSA, como organismo de promoción, participa con frecuencia en las principales ferias del vino alrededor del mundo con el fin de potenciar y exhibir el enoturismo y los productos vinícolas sudafricanos. Para que esto suceda, WOSA trabaja estrechamente con las autoridades turísticas locales, con el objetivo de mantenerse informada de los cambios y acontecimientos que van sucediendo en los diferentes destinos.

WOSA es muy activa en la creación de - y participación en - diferentes ferias, roadshows, seminarios y conferencias de prensa a nivel nacional e internacional. Su principal evento es Cape Wine que tiene lugar cada tres años en Ciudad del Cabo. Se considera como el acontecimiento comercial "insignia" de la industria vitivinícola sudafricana (WOSA, 2015).

Tomando como base la experiencia enoturística impulsada por WOSA, se han establecido en Sudáfrica otras estructuras de OGD/DMO de carácter regional. Uno de los casos de mayor éxito es el de Stellenbosch Wine Routes, entidad enfocada en los diversos productos enoturísticos que se pueden encontrar en el área de Stellenbosch, como parte de la región global Cape Wineland.

Este OGD/DMO representa a más de 200 productores de vino dentro del área geográfica de Stellenbosch. En su sitio web muestran información sobre los eventos que tendrán lugar en torno a la cultura del vino, así como consejos para el viajero con respecto a degustaciones de vinos, atracciones y alojamiento. Se especializa también en recorridos, mediante el diseño y comercialización de 5 rutas enoturísticas que muestran al enoturista la diversidad y complementariedad que puede descubrir y experimentar en esta zona del país.

A través de su sitio web favorece la posibilidad de individualizar la búsqueda del enoturista mediante diferentes filtros de elevada segmentación: niños, discapacitados, fines de semana, etc. Como resultado de esta búsqueda, el cliente tiene acceso a un mapa detallado con todas las bodegas que se pueden encontrar a lo largo de la ruta, así como información más técnica (variedades de uva, cultivo, etc).

En cuanto a los diferentes actores que participan en esta estructura de OGD, se incluyen los siguientes

- Sector privado: Representado por distintas entidades, tanto en el ámbito de los productores vinícolas sudafricanos de la región de Stellenbosch, como de otros tipos de empresas turísticas complementarias (restaurantes, alojamientos...), que favorecen la experiencia global del viajero.

- Organismos gubernamentales: El Ministerio de Turismo a través de sus entidades locales, en este caso, la Oficina de Turismo Local del Distrito de Cape Winelands, que participa activamente en el impulso de acciones concretas (organización y promoción de ferias, eventos, etc.) contribuyendo a paliar la estacionalidad y garantizando flujos turísticos en temporada baja.

- Red internacional: Como parte de los vinos de Cape Winelands, las Rutas del Vino de Stellenbosch forman parte de las seis atracciones turísticas más populares de Sudáfrica y también están conectadas a la red global Great Wine Capitals Network.

- Viajeros / clientes: La opción de voto en TripAdvisor, demuestra que las Rutas del Vino Stellenbosch son populares entre la comunidad de turistas que viajan a esa zona en Sudáfrica. 
Se puede concluir así que en Sudáfrica la unión de diferentes actores mediante el denominado "enfoque colectivo del enoturismo" es clave para el éxito global de un destino. En los dos casos descritos, una alianza público-privada ha conseguido funcionar de común acuerdo en objetivos y estrategias alineadas, y ello ha permitido transmitir un mensaje creíble y único a nivel nacional e internacional. Otra de las claves de éxito más importante es que los diferentes OGDs tienen apoyo nacional en materia de comunicación. De hecho, el enoturismo se presenta como un elemento clave para el desarrollo estratégico del turismo y los pilares promocionales de Sudáfrica y como uno de los principales atractivos de la marca-país (Destinate News, 2016).

\subsection{El Condado de Sonoma: Un caso de éxito en Estados Unidos}

El Condado de Sonoma es un distrito de 485.000 habitantes, ubicado en la costa del Pacífico, en el estado de California, a 45 minutos de San Francisco. Es visitado por más de 7,4 millones de turistas anuales, que gastaron más de mil millones de dólares en 2006. La industria hotelera y turística en el Condado de Sonoma tiene así un impacto económico enorme y mantiene 19.000 empleos, siendo pieza clave de la economía local (Sonoma County Tourism, 2016).

A partir de esta realidad, y mediante un proceso colaborativo público-privado con el objetivo de desarrollar el turismo en el condado, se constituyó el Sonoma County Tourism (SCT), como organización oficial de marketing de destino local. SCT es una organización privada y sin ánimo de lucro cuyo principal objetivo es incrementar el número de pernoctaciones en el condado de Sonoma, California. El órgano de gobierno de SCT está compuesto por un consejo de directores voluntario, que cuenta con las contribuciones de los comités, de las partes interesadas y del público en general (Sonoma County Tourism, 2016). SCT supone un modelo de referencia y éxito en gestión pública/privada de un OGD/ DMO, por lo que un breve análisis de los antecedentes y el proceso de creación de esta estructura parece relevante. La idea original de SCT surge en marzo de 2001, cuando los principales stakeholders de la industria turística y del sector público comenzaron a plantear conjuntamente nuevas formas de crear una estructura real y operativa de DMO, para promover el turismo en el condado a través de un plan de marketing. La Asociación de Alojamiento del Condado de Sonoma (SCLA, por sus siglas en inglés) se convirtió en líder de este proyecto, fomentando una propuesta de DMO basada en la cooperación entre entidades públicas y actores privados, e incluso involucrando a la comunidad local.

En noviembre de 2004, la Junta de Asesores del Condado estableció el Área de Mejora de Negocios Turísticos del Condado de Sonoma (SCTBIA), que entró en vigor en 2005, y que creó el marco para un Plan de Marketing más organizado, que finalmente se materializó a través de la Oficina de Turismo del Condado de Sonoma (SCTB).

Meses después, en junio de 2005, la SCTB firmó un contrato con la Junta de Asesores del Condado para promover las visitas con pernoctación incluida, i.e., el turismo en el condado, convirtiéndose así en el DMO oficial. En el año 2012 la región se consolidó como destino enoturístico y se desarrolló otra iniciativa de éxito a través de la estrategia de marca con Sonoma County Vintners y Condado de Sonoma Winegrape Commission. Según Fischang, Presidente y CEO de Sonoma County Tourism: "Básicamente, los cultivadores cultivan las uvas, los viticultores hacen el vino, y el DMO trae gente a Sonoma para beber el vino“ (Destination Marketing Association International, 2015, 07.08). Se trata sin duda de una exitosa iniciativa basada en una suma de sinergias entre los distintos actores que participan en el destino enoturístico, en la misma entrevista continua explicando su método: "Formamos un grupo llamado Consejo del Presidente, con líderes electos, líderes clave en turismo, vinicultores, viticultores y cada una de las 15 zonas vitivinícolas del condado de Sonoma, que ha tenido un gran impacto en la comunidad local".

Otro de los logros de esta DMO es que ha podido impulsar una ley para el etiquetado conjunto, de manera que cada botella de vino del Condado de Sonoma "garantiza" estar producida al menos con el $75 \%$ de uvas del condado.

Asimismo, el SCT creó una iniciativa de promoción denominada "Sonoma in the City", de manera que diferentes actores de la DMO y algunos viticultores viajan con sus productos a las ciudades que representan sus principales mercados domésticos.

Otro gran evento de promoción conjunto se produjo en 2016, cuando SCT y sus dos socios (Sonoma County Vintners y Sonoma County Wine grape) formaron parte de los patrocinadores oficiales de la "SuperBowl 50" en San Francisco. Fischang ya adelantó en 2015 que todo el vino que se serviría en dicho evento sería de Sonoma (Sonoma Wine Grape, 2015). 
La gestión de destinos requiere la colaboración en torno a una visión y estrategias comunes para tener éxito. Es importante compartir las mejores prácticas y seguir buscando acciones de mejora en un proceso permanente.

\section{Enoturismo en España: el estado de la cuestion}

Es en la década de 1990, coincidiendo con la simbiosis conceptual entre vino y turismo -potenciada en gran medida en mercados del Nuevo Mundo como Australia y Nueva Zelanda (Fraser y Alonso, 2006; López-Guzmán, 2013) - cuando nace en España una incipiente actividad enoturística.

De forma paralela a lo acontecido a nivel internacional, es en esta misma época cuando el consumo de vino en España decrece de forma alarmante en beneficio de la cerveza (Alvira, 1986). Frente a esta situación, los productores vinícolas reaccionan con un doble enfoque estratégico a nivel comercial. Por un lado apuestan por la exportación, llegando a lograr un liderazgo mundial para España en términos de volumen (OIV, 2017). Por otro lado, incursionan en un tímido y primitivo concepto de enoturismo, mediante una adecuación básica de las instalaciones de las bodegas para la recepción de visitantes, financiada en gran medida por las ayudas europeas a las industrias rurales surgidas a raíz del ingreso de España en la Unión Europea en 1986 (Saraceno, 2007).

No es sino ya en 1994 (López-Guzmán et al, 2013) cuando se crea la Asociación Española del Ciudades del Vino (Acevin), con el objetivo de aportar un instrumento que fomentase la competitividad de la industria vitivinícola apoyada en herramientas estratégicas y sectoriales diversas, entre las que se encuentra el turismo, y articulada mediante un elemento geográfico - las ciudades del vino - con problemáticas y necesidades comunes.

Durante estas etapas nacientes, el enoturismo en España se limita en gran medida a la oferta de productos turísticos relativamente estáticos, y principalmente tangibles, desarrollados en torno a la concepción de la bodega como recurso físico y a la cata como actividad principal. Comienza a surgir además, en línea con las tendencias europeas del momento, una incipiente conceptualización de ruta del vino como propuesta de producto turístico integrador y dinámico en sus variables geográfica y de composición de la oferta.

La complejidad estratégica y operativa de las rutas del vino requiere sin embargo una coordinada colaboración público-privada, que se materializa con el lanzamiento en 2001 del "Club de Producto Rutas del Vino", con el apoyo de los Ministerios de Economía y de Agricultura, Pesca y Alimentación. Las Rutas del Vino de España se conceptualizan por iniciativa pública como una de las acciones ejecutoras del programa "Calidad en los productos turísticos", uno de los diez programas que componían los ejes estratégicos del Plan Integral de Calidad del Turismo Español (PICTE) 2000-2006, elaborado por la Secretaría de Estado de Turismo del Ministerio de Economía (Secretaria de Estado de Comercio, Turismo y de la Pequeña y Mediana Empresa, 1999) y que tenía por objeto el desarrollo de productos de calidad que contribuyeran a la diversificación y desestacionalización de la actividad turística en España.

Es en base a los datos extraídos de los establecimientos (bodegas y museos) adheridos a este sistema de rutas, que el mercado turístico español simplifica y extrapola estos resultados al conjunto del enoturismo nacional, posiblemente por la carencia de otras fuentes de datos agregados, si bien esta práctica asume por tanto una metodología que no corresponde fielmente a la realidad del subsector enoturístico español en su globalidad.

Cabe mencionar por tanto que, ni la Secretaría General de Turismo de España, ni en última instancia la Organización Mundial de Turismo, publican todavía estadísticas oficiales sobre enoturismo en España (o en el mundo), por lo que se debe recurrir inevitablemente a los datos parciales del Observatorio Turístico Rutas del Vino de España (Acevin, 2017). Se desprende de dicho informe que el número de enoturistas (enovisitantes en términos estrictos) en las Rutas del Vino de España en 2016 fue de 2,7 millones. Ello supone un aumento en términos absolutos del $21 \%$ respecto al año anterior, si bien la cifra está distorsionada por el incremento del número de establecimientos adheridos a las Rutas del Vino (del 4,8\% respecto a 2015; 28 unidades de negocio adicionales en total). En realidad, si se compara la cifra de 2,7 millones de enovisitantes con los 242,0 millones de movimientos turísticos totales de residentes y no-residentes en 2016 en España (INE, 2017a; INE, 2017b), resulta que solo un 1.1\% visitaron al menos una instalación enoturística, En cuanto al gasto medio del enoturista por bodega, es inferior a 25 euros por visitante, con algo menos de 8 euros por lo que se considera "visita estándar a bodega" - que incluye la visita guiada y la degustación - y hasta 17 euros adicionales correspondientes al desembolso medio del visitante en tienda. 
Del total de "enoturistas" registrados, una amplia mayoría del 77\% es de procedencia nacional, mientras que el $23 \%$ restante tiene procedencia internacional. Por otra parte, en lo que se refiere a la distribución temporal de las visitas, son los meses de primavera y otoño los que más actividad registran.

Estos datos contrastan sin duda con la realidad vitivinícola del país, ya que España se posiciona como la superficie de viñedo más importante a nivel mundial y el tercer productor de vino del mundo, solo por detrás de Italia y Francia (OIV, 2017). A mayor abundamiento, estableciendo un análisis cruzado de correlación entre datos vitivinícolas y valores enoturísticos de los principales mercados competidores, hay que destacar que Italia recibe actualmente 14 millones de enoturistas según el Observatorio sobre el Turismo del Vino de la Associazione Nazionale Città del Vino (2017). Por su parte Francia, que produce tan sólo un $10 \%$ más de vino que España, recibe muchos más enoturistas, habiendo alcanzado los 10 millones en 2016 (VFW, 2017). Además, en el caso de Francia, el porcentaje de distribución de visitantes atendiendo a su procedencia está muy equilibrado, correspondiendo un $58 \%$ a franceses y el restante $42 \%$ a enoturistas internacionales.

Un estudio de la Organización Mundial del Turismo señala que, por su parte, Estados Unidos contó en 2014 con más de 15 millones de turistas que se movieron fundamentalmente por motivos enoturísticos. Ese estudio (OMT, 2016b: 8) concluía que "en el caso español (...) el apoyo institucional es menor que en los destinos competidores y tiene poca influencia como destino entre los líderes de opinión”.

Los datos analizados ponen de manifiesto carencias y oportunidades en el sistema enoturístico español. En primera instancia se hace necesaria la adopción de medidas estructurales que contribuyan al posicionamiento del enoturismo como uno de los ejes prioritarios de la estrategia de desarrollo turístico nacional, ya no sólo a nivel de política turística, sino como herramienta de consolidación de la marca-país.

Para ello parece lógico aprovechar la coyuntura del mercado vitivinícola existente, como recurso sobre el que consolidar una alternativa turística que aporte diversificación, calidad, desestacionalización, prestigio, y que según promulga la propia Organización Mundial del Turismo (2016a), puede contribuir a promover el turismo sostenible, jugando un rol importante en la preservación de los recursos naturales y culturales del destino, promoviendo enfoques innovadores en la concepción de la experiencia turística, y contribuyendo al desarrollo turístico de las áreas rurales.

Sin embargo, para dotar de competitividad y calidad al creciente sector enoturístico español, no se puede prescindir de la adopción de un sistema de gestión estratégica articulado mediante entes de gestión colaborativa (OGDs/DMOs), que se conviertan en una plataforma dinamizadora a nivel estratégico y operacional del sector.

Pero en este contexto de retos y carencias institucionales, tal como apuntaba la OMT, están surgiendo también iniciativas que plantean oportunidades en la consolidación de actores clave. Así, en 2015 surge la Asociación Española de Enoturismo (AEE), un organismo privado creado para favorecer la necesaria colaboración entre establecimientos empresariales, asociaciones y entidades públicas en torno a una marca global, Enoturismo de España, que contribuya al posicionamiento y éxito del conjunto de estas actividades empresariales y públicas, tanto a nivel nacional como internacional.

Actualmente la AEE cuenta con más de 200 establecimientos miembros en todo el territorio nacional que forman parte de los diversos eslabones de la cadena de valor enoturística, estableciendo una representación homogénea en materia de posicionamiento, promoción y visibilidad turística, especialmente a nivel internacional, y constituyendo así un elemento potenciador de la coopetitividad entre los establecimientos y destinos.

El enoturismo en España aglutina una serie de esfuerzos e iniciativas privadas que conviven fuera de los sistemas de rutas, y la AEE considera necesario construir una estrategia nacional de colaboración público-privada y con la sociedad civil que asuma la coordinación de los distintos niveles de planificación estratégica (AEE, 2017): desde la generación de sistemas de información para la inteligencia de los mercados enoturísticos y la implementación de acciones de gestión del conocimiento en un marco de política turística (Fayos-Solà, Fuentes y Muñoz, 2012b), a la promoción de la excelencia y calidad en el conjunto de la cadena de valor, y la consolidación de los canales de comercialización y promoción en mercados internacionales y nacionales.

\section{Conclusiones y recomendaciones}

El enoturismo supone en la actualidad una muy importante actividad empresarial, cultural y social en el amplio abanico de una oferta turística híper-segmentada, con potencial para contribuir a los objetivos estratégicos de los destinos que lo promueven: Desarrollo económico y social con creación de riqueza, 
capital humano, y calidad de vida, pasando por la preservación del patrimonio tangible e intangible, mejora del potencial emprendedor, de la calidad y competitividad, innovación, generación de sinergias colaborativas y marcos institucionales para la gobernanza participativa..

Para conseguir que las potencialidades que conlleva la integración del enoturismo en el destino se implementen efectivamente, es preciso crear herramientas eficaces y eficientes de gestión de destinos turísticos. Tal como se ha venido demostrando internacionalmente en muy diversos ámbitos de la actividad turística, los Organismos de Gestión de Destinos (OGDs) constituyen una iniciativa institucional estratégica para garantizar una eficiente coordinación entre agentes, objetivos, y programas de acción en un destino.

El enoturismo no es una excepción a esta tendencia, y las diversas prácticas aquí analizadas, tanto en el Nuevo como en el Viejo Mundo, ponen de manifiesto la conveniencia de estructurar de forma coherente los diversos elementos estratégicos, como medio para alcanzar la competitividad de los destinos enoturísticos.

Queda patente asimismo, a través de las diferentes aproximaciones efectuadas en este artículo, que el enoturismo en España está todavía en proceso de alineación con las tendencias internacionales. La falta de una gestión del conocimiento específica, la inexistencia de un marco de gobernanza de destinos enoturísticos propiamente dicho, o la carencia de un observatorio nacional que genere datos de relevancia operativa, ponen de manifiesto la importancia de la tarea por hacer.

En este contexto, y desde una perspectiva multiangular de la misma realidad, hay que hacer una primera observación básica: Los actores del turismo del vino deben trabajar juntos para crear una cultura, un estilo de trabajo, una imagen y un posicionamiento común para los destinos enoturísticos.

A partir de la evaluación comparativa de las mejores prácticas analizadas y de la realidad actual del enoturismo en España, se presenta una serie de propuestas y recomendaciones estratégicas para la gestión de DMOs enoturísticos en el caso español.

Se recomienda promover un sistema de gestión colaborativa del enoturismo en España, que integre la globalidad de los actores involucrados en todos los eslabones de la cadena de valor, representando al sector y concediéndole una voz homogénea a nivel internacional y nacional.

En este contexto, es clave la creación de una plataforma dinamizadora y operacional del sector enoturístico en España, conformando el organismo de referencia para los diversos agentes implicados (enoturistas, proveedores, productores, administración nacional, regional, local, etc.).

Se hace necesario asimismo fomentar la relación entre los diversos elementos productivos y de servicios ligados a la cadena vitivinícola-enoturística en España, promoviendo sinergias que contribuyan a la proyección de una marca "Enoturismo de España", que integre los esfuerzos individuales de los actores involucrados.

Para una adecuada gestión integral del enoturismo, hay que desarrollar programas que cubran todas las fases de la planificación estratégica, hacia los objetivos de calidad y competitividad del sector enoturístico en España. Asimismo es clave generar sistemas de información para la inteligencia de los mercados enoturísticos, analizando la realidad del subsector enoturístico español en su globalidad y generando datos operativos comparables a los disponibles en mercados internacionales competidores que faciliten la toma de decisiones estratégicas.

Por último, pero de gran importancia, hay que implementar acciones de gestión del conocimiento que promuevan (i) la investigación y análisis; (ii) la difusión de conocimiento a través de programas de formación y capacitación de alta calidad, y (iii) la aplicación del conocimiento mediante la creación de marcos regulatorios y normativos (voluntarios o no) y la innovación como elemento dinámico de aplicabilidad transversal.

Estas acciones establecerán bases para la coordinación entre los principales actores de los sectores público, privado y civil involucrados en la cadena de valor del enoturismo, en torno a una visión, misión y estrategia compartidas. La figura institucional de Organismos de Gestión de Destinos (enoturísticos) es el marco de acción óptimo para todos los interesados, generando capacidad para aunar acciones y asegurar la calidad y competitividad, a nivel internacional y nacional, de los destinos enoturísticos de España.

\section{Bibliografia}

Acevin - Asociación Española de Ciudades del Vino, 2017. Informe de visitantes a bodegas y museos del vino asociado a las Rutas del Vino de España - Año 2016. 
AEE - Asociación Española de Enoturismo,

2017. Disponible en: https://www.enoturismodeespaña.es/web/ [Acceso: 12/02/2017].

AEE - Asociación Española de Enoturismo,

2015. Disponible en https://www.enoturismodeespaña.es/web/text.php?id section=1467 [Acceso 23/01/2017].

Alebaki, M. y Iakovidou, O.

2011. Market Segmentation in Wine Tourism: A comparison of approaches. An International Multidisciplinary Journal of Tourism. 6(1): 123-140.

Alvira Martín, F.

1986. Cambios en el consumo de bebidas alcohólicas en España. Reis: Revista española de investigaciones sociológicas 34: 111-130.

Birch, S.

2007. Wine Tourism: An efficient and profitable channel of distribution [Conferencia].En Vinexpo Bordeaux. WineTourism: an efficient and profitable channel of distribution. VIinexpo, Bordeaux, 19-6-2007. [Recuperado de nota de prensa accesible en http://www.wine.co.za/news/news. aspx?NEWSID $=10322 \&$ Source $=$ News] .

Cabello, S.A., y Pascual, N.

2015. La construcción del turismo en nuevos destinos: Luces y sombras. El caso de La Rioja (España). Revista de Ciencias Sociales y Humanidades, 24: 30-48.

Carlsen, J. y Charters, S.

2006. Conclusion: The future of wine tourism research, management and marketing. En J. Carlsen y S. Charters (Eds.) Global Wine Tourism: Research Management and Marketing, London: CABI Publishing.

Charters, S. y Carlsen, J.

2006. Introduction. En J. Carlsen y S. Charters (Eds.) Global Wine Tourism: Research Management and Marketing, London: CABI Publishing.

Christou, E. y Nella, A.

2012. Web 2.0 and Networks in Wine Tourism: The Case Studies of greatwinecapitals.com and wineandhospitalitynetwork.com. En Sigala, M., Christou, E. y Gretzel, U. (Eds.) (2012), Social Media in Travel, Tourism and Hospitality: Theory, Practice and Cases. Surrey: Ashgate Publishing.

Cosa, M.

2010. Gestión y estructura organizativa de las Destination Management Organizations: Un enfoque económico-empresarial. Universidad de Bari, Italia

Destination Marketing Association International

2015. How Sonoma County Changed Destination Marketing. [Blog, 07-08-2015. Recuperado de http:// www.destinationmarketing.org/blog/how-sonoma-county-changed-destination-marketing] .

Destinate News

2016. Wine Tourism to take center stage in the marketing of South Africa. [Blog. Recuperado el 19 de julio de 2016 de http://www.destinate.co.za/blog/entry/wine-tourism-to-take-center-stage-in-the-marketing-of-south-africa\#.WMpXTTvhDIV].

Díaz Armas, R.J.

2008. Potencialidad e integración del "turismo del vino" en un destino de sol y playa: El caso de Tenerife, Pasos - Revista de Turismo y Patrimonio Cultural 6(2): 199-212.

Domenach, Audrey

2016. Les chiffres-clés du marché de l'œnotourisme en France. Viti Leaders/Mon Viti, 23/02/2016.

[Recuperado de https://www.mon-viti.com/articles/commercialisation/les-chiffres-cles-du-marche-de-loenotourisme-en-france].

Elías Pastor, L. V.

2006. El turismo del vino. Otra experiencia del ocio. Bilbao, Universidad de Deusto.

Fayos-Solà, E., Alvarez, M. D. y Cooper, C. (Eds.)

2014. Tourism as an Instrument for Development: A Theoretical and Practical Study, Londres, R.U. Emerald Group Publishing Limited.

Fayos-Solà, E., Ruhanen, L., de Bruyn, C., Muñoz, A., Fuentes, L. y Fernández, A.

2012a. Conclusion: Understanding and Enacting the Strategic Role of Tourism. En E. Fayos-Solà, J. Matos de Silva, y J. Jafari (Eds.) Knowledge Management in Tourism: Policy and Governance Applications, pp.317-27. Londres, R.U.: Emerald Group Publishing Limited.

Fayos-Solà, E., Fuentes Moraleda, L. y Muñoz-Mazón, A.

2012. Elaborando un plan de política turística: consideraciones metodológicas. Papers de Turisme 51: 41-65. 
Fayos-Solà, E. García, P. y Moreda, A.

2002. A Modest Proposal on the Tourism Policy and Destination Management Research Programme.

UNWTO TedQual 5: 21-25.

FEV - Federación Española del Vino (2017), Memoria 2016, FEV.

Fraser, R. A. y Alonso, A.

2006. Do Tourism and Wine Always Fit Together? A Consideration of Business Motivations. En Carlsen, J. y Charters, S. (Eds.)

2008. Global Wine Tourism: Research, Management and Marketing (pp.19-26), Cambridge: Cabi International.

Gatti, S., Giraud-Héraud, E., y Mili, S. (Eds.)

2003. Wine in the Old World, Franco Angeli, Milano, Italy.

Herrero, M. D.

2014. Diseño y desarrollo de un sistema de Gestión de Calidad para MIPYMES turísticas Centroamericanas (Tesis de doctorado). Universidad Rey Juan Carlos, Madrid (España).

INE - Instituto Nacional de Estadística de España

2017. Encuesta de Turismo de Residentes (ETR/FAMILITUR) 2016 http://www.ine.es/daco/daco42/etr/ etr0416.pdf [Acceso 29/03/2017].

INE - Instituto Nacional de Estadística de España

2017. Estadística de Movimientos Turísticos en Fronteras (FRONTUR) 2016 http://www.ine.es/daco/ daco42/frontur/frontur1216.pdf [Acceso 31/01/2017].

López-Guzmán, T. J., Rodríguez-García, J. y Vieira-Rodríguez, A.

2013. Revisión de la literatura científica sobre enoturismo en España. Cuadernos de Turismo 32(1):171-188.

López-Guzmán, T. J. y Sánchez, S. Ma .

2008. La creación de productos turísticos utilizando rutas enológicas. Pasos - Revista de Turismo y Patrimonio Cultural, 6 (2): 159-171.

López Sánchez, J. A.

2010. Posibilidades de desarrollo del enoturismo en la Denominación de origen Jerez-Xerry-Sherry y Manzanilla de Sanlúcar de Barrameda y Vinagre de Jerez. Boletín de la Asociación de Geógrafos Españoles 53: 21-41.

Loubser, S.

2004. Wine tourism needs holistic approach. Wineland, Bussines and Marketing.1, jun. 2004. [Recuperado de http://www.wineland.co.za/wine-tourism-needs-holistic-approach/].

Maduro, A. V., Guerreiro, A., Oliveira, A.

2015. O turismo industrial como potenciador do desenvolvimento local - estudo de caso do Museu do Vinho de Alcobaça em Portugal. Pasos - Revista de Turismo y Patrimonio Cultural, 13 (5), 1129-1143. Morgenthal, A.

2015. Round table discussion: wine tourism strategy. En Bonet, J.L. (Ponente).Wine Tourism Strategy. Conferencia llevada a cabo en el congreso Wine Tourism, a strategy for everyone, Logroño, 9-10 noviembre 2015.

Muñoz-Mazón, A. y Velasco, M.

2015. Colaboración y gobernanza para el desarrollo turístico. Aranjuez como estudio de caso. Cuadernos de Turismo, 35: 311-334.

Observatorio sobre el Turismo del Vino de la Associazione Nazionale Città del Vino

2017. Disponible en http://www.cittadelvino.it/articolo.php?id=MjMwMQ== [Acceso 12/02/2017].

OEMV - Observatorio Español del Mercado del Vino

2017 . Informe: Balance vitivinícola. Campaña 2015/2016, Madrid: OEMV.

OEMV - Observatorio Español del Mercado del Vino

2017b. Informe: Superficie de viñedo en España 2016, Madrid: OEMV.

OEMV - Observatorio Español del Mercado del Vino

2017. Informe: Número de bodegas en España - 01.01.2017, Madrid: OEMV.

OIV - International Organisation of Vine and Wine

2017. 2017 World Vitiviniculture Situation OIV Statistical Report on World Vitiviniculture, Paris: OIV.

OMT - Organización Mundial del Turismo

2016. Georgia Declaration on Wine Tourism, Madrid: OMT.

OMT - Organización Mundial del Turismo

2016b. Prototipo de turismo enológico de la OMT. UNWTO Wine Tourism Prototipe. Madrid: OMT. 
OMT - Organización Mundial del Turismo

2007. A Practical Guide to Tourism Destination Management. Madrid: OMT.

Ramis Hernández, A.

2010. Turismo y vino en el mundo. El caso de bodegas Enrique Mendoza (Proyecto Fin de Máster).

Universidad de Alicante, España. [Disponible en web http://rua.ua.es/dspace/bitstream/10045/15168/1/

Turismo\%20y\%20vino\%20en\%20el\%20mundo_Ramis.pdf].

Recevin (Red Europea de Ciudades del Vino)

2006. Vademecum del Enoturismo Europeo [ProyectoVINTUR-El espacio europeo del enoturismo] Recuperado de [http://www.recevin.net/userfiles/file/VINTUR/VADEMECUM\%20ENOTURISMO\%20 EUROPEO\%20ES.pdf].

Saraceno, E.

2007. Políticas rurales de la Unión Europea y proyectos territoriales de identidad cultural. Revista Opera 7: 167-189.

Secretaria de Estado de Comercio, Turismo y de la Pequeña y Mediana Empresa

1999. Plan Integral de Calidad del Turismo Español (2000-2006). Ministerio de Economía y Hacienda. <http://www.mcx.es/turismo/picte2000> [Acceso: 12/12/2016].

Sheehan, L., Vargas-Sánchez, A., Presenza, A. y Abbate, T.

2016. The Use of Intelligence in Tourism Destination Management: An Emerging Role for DMOs. International Journal of Tourism Reseach, 18 (6): 549-557.

Sigala, M., Christou, E. y Gretzel, U. (Eds.)

2012. Social Media in Travel, Tourism and Hospitality: Theory, Practice and Cases. Surrey: Ashgate Publishing.

Sonoma County Tourism

2016. About Sonoma County Tourism. Recuperado de http://www.sonomacounty.com/about-sonoma-county-tourism [Acceso el 20-02-2017].

Sonoma Wine Grape

2015. Super Bowl 50 Host Committee Partnership. Recuperado de www.sonomawinegrape.org: http:// www.sonomawinegrape.org/SuperBowl50

Vázquez de la Torre, G.M. y Melián, A.

2008. Rutas turísticas enológicas y desarrollo rural. El caso estudio de la denominación de origen Montilla-Moriles en la Provincia de Córdoba. Papeles de Geografía 47-48: 159-170.

VFW (Visit French Wine)

2017. Wine Tourism in France in 2016, VFW. Acceso https://www.visitfrenchwine.com/en/product/ wine-tourism-france-numbers el 20-02-2017.

Viator Blog: tours and activities around the world.

2013. Top 10 Wine Destinations Around the World. [Blog. Recuperado de http://www.huffingtonpost. com/viator/top-10-wine-destinations_b_3876855.html].

Williams, P. W., Graham, K. y Mathias L.

2006. Land Use Policy and Wine Tourism Development in North America's Pacific Northwest. En J. Carlsen y S. Charters (Eds.) Global Wine Tourism: Research Management and Marketing, London: CABI Publishing.

WOSA - Wines of South Africa

2015. Cape Wine [International wine trade show]. 15-17 septiembre, 2015. Cape Town

International Convention Centre. Ciudad del Cabo. [Acceso en http://www.capewine2015.com/ el 20-02-2017]. 